Mots. Les langages du politique

Maurice Tournier, Des sources du sens. Propos d'étymologie sociale 3

\title{
Éléonore Yasri-Labrique
}

\section{(2) OpenEdition \\ Journals}

Édition électronique

URL : https://journals.openedition.org/mots/1266

DOI : $10.4000 /$ mots. 1266

ISSN : 1960-6001

Éditeur

ENS Éditions

\section{Édition imprimée}

Date de publication : 1 novembre 2007

Pagination : 120-122

ISBN : 978-2-84788-113-4

ISSN : 0243-6450

Référence électronique

Éléonore Yasri-Labrique, " Maurice Tournier, Des sources du sens. Propos d'étymologie sociale 3 », Mots. Les langages du politique [En ligne], 85 | 2007, mis en ligne le 01 novembre 2009, consulté le 23 avril 2022. URL : http://journals.openedition.org/mots/1266 ; DOI : https://doi.org/10.4000/mots. 1266 


\section{Des sources du sens. Propos d'étymologie sociale 3}

Maurice Tournier

2002, Lyon, ENS Éditions, Collection «Langages», 308 p.

Après Des mots sur la grève. Propos d'étymologie sociale 1 (première édition: 1993) et Des mots en politique. Propos d'étymologie sociale 2 (première édition : 1997), Maurice Tournier poursuit son exploration linguistique de la signification en réunissant une série d'analyses denses et passionnantes mettant en valeur les sources sociohistoriques et / ou textuelles du sens. Des sources $d u$ sens. Propos d'étymologie sociale 3 se présente en effet comme un recueil de 22 contributions, publiées pour la plupart entre 1990 et 2002 et reprises dans un ensemble cohérent de réflexions variées sur le langage en politique et les aspects politiques du langage.

L'ouvrage s'ouvre sur un «Avant-propos » où l'auteur présente brièvement les trois modes d'accès au sens : personnel et donc « aussi inconnaissable que nous-mêmes» (p. 9), historique et textuel. Ce sont les deux derniers qui sont au cœur du livre, dans une perspective à la fois lexicologique et sociologique où se rejoignent, d'une part, la sociohistoire et d'autre part, l'analyse du discours, avec, dans certains articles, la mise en œuvre de méthodes d'investigation statistique de la textualité de surface regroupées sous le nom de "lexicométrie politique» (p. 10). Deux études plus longues et plus ambitieuses, significatives des enjeux de l'étymologie sociale dont le «projet central est de questionner l'implantation sociale des mots» (p. 17), encadrent le recueil: "L'étymologie sociale face au discours politique» (p. 11-46) et «Histoire résumée de la "langue française" " (p. 279-300) proposent non seulement des définitions argumentées de notions cruciales telles que le champ politique, le sens, le discours, la langue..., dans un foisonnement d'exemples et de références transdisciplinaires, mais également une interrogation approfondie sur les interactions entre le langage et le politique dans leurs aspects diachroniques et synchroniques.

Les vingt autres études s'offrent au lecteur sans ordre apparent - ni chronologique, ni méthodologique, ni thématique. Cette absence de classement, délibérée, pourrait en perturber certains; elle correspond en fait à un désir de liberté que l'auteur revendique pour lui comme pour l'autre. La pensée de Maurice Tournier ne se veut prisonnière d'aucun carcan; elle s'appuie sur les mots qu'elle explore pour dévoiler sans tabou les richesses insoupçonnées des signes énoncés jour après jour au cours des siècles, richesses des origines, des évolutions, des interprétations, des contextualisations..., et laisse entrevoir un trésor infini que chacun est invité à s'approprier sans retenue. Du coup, le ton, volontairement polémique, ne permet pas une lecture terne ou indifférente. Ce jaillissement d'idées, tout à la fois spontané et érudit, provoque mille questions et réactions grâce auxquelles le lecteur, ancré dans une actualité déjà réinvestie par l'auteur, devient également acteur : on ne peut s'empêcher de mettre en relation 
les thèmes abordés par Maurice Tournier avec le monde signifiant qui nous entoure et de continuer, une fois le livre refermé, la recherche qu'il a initiée.

Ainsi, la chronique intitulée "Colonie, entre le droit, les mythes et les usages», publiée d'abord en 1997, s'épaissit d'une résonance particulière dix ans plus tard, au moment où reparaît le débat sur les «bienfaits de la colonisation » et où les mots se chargent davantage encore de significations multiples dans les échanges où la parole se trouve directement en lien avec des mémoires blessées. De même, la question «Europe, "fille d'Asie", "métisse" d'origine, qui embrasseras-tu?» (p. 89), conclusion du texte «Europe, terme et terred'immigration », daté de juillet 2000, s'entoure en France d'échos de plus en plus sonores et insistants au fil des campagnes électorales de ce début de millénaire, et ce notamment avec l'ouverture contestée des négociations officielles avec la Turquie, pays eurasiatique, dans le cadre de son éventuelle adhésion à l'Union européenne.

Le sens et la portée des mots en politique, leur banalisation ou leur détournement, leur cheminement de la nomination à l'insulte, de la désignation à la récupération, sont aussi au centre de chroniques comme "Les mots fascistes, du populisme à la dénazification» (juin 1998) ou encore «Race, populisme, Shoah, mots d'hier, périls de demain» (octobre 1998) : examinées avec méthode à la lumière de l'histoire et de l'analyse de discours, les significations passées et actuelles sont décrites, appréhendées, décortiquées puis restituées au public par l'auteur qui apporte ainsi des éclairages intéressants non seulement pour l'historien ou le linguiste, mais aussi pour le sociologue et le citoyen. L'homme engagé n'est d'ailleurs jamais loin. Ainsi déclare-t-il: «Si nous ne parlons pas, nous chercheurs en vocabulaire, des problèmes que pose l'émergence du Front national et, de façon plus large, de celle d'un mouvement raciste en France, d'autres le feront en d'autres termes» (p.195), rejoignant la position du chercheur suisse Uli Windisch : «À quoi servent les connaissances des sciences humaines et sociales si elles ne sont pas aussi injectées dans le débat et l'action politiques?» (Stéréotypage, stéréotypes: fonctionnements ordinaires et mises en scène, t. 2, Paris, L'Harmattan, 2007, p. 312).

On retiendra également les nombreuses études du discours syndical ou politique, généralement basées sur des enquêtes lexicométriques révélatrices, et, plus léger mais tout aussi sérieux, le clin d'œil amusé, précis et pertinent à nos aïeules et nos contemporaines dans « Madame la marquise » qui, à travers l'étude des noms de professions au féminin, pan du vocabulaire français souvent en errance, interroge sur la reconnaissance de la place des femmes dans la société.

Certes, ce recueil de contributions proposé par Maurice Tournier n'est pas exhaustif. II n'en a d'ailleurs pas la prétention. Mais les grandes préoccupations du moment, de l'écologie à l'identité nationale en passant par la francophonie, sont replacées dans leur contexte sociohistorique et intertextuel, ce qui permet au lecteur, averti ou non, d'échapper aux évidences apparentes de l'air du 
temps, aux interprétations ponctuelles données pour des vérités immuables, et de se replonger dans l'aventure du sens en intégrant l'idée essentielle selon laquelle «il n'y a rien de linéaire dans une évolution sémantique » (p. 83) : «Les mots n'ont pas de définition en soi» (p. 196). La signification est fonction de rapports complexes entre les pratiques sociales ou individuelles et les occurrences des signes énoncés pour les évoquer. D’où un questionnement récurrent dans l'ouvrage, mais en particulier dans « Des dictionnaires de langue aux inventaires d'usages», du rôle des «dictionnaires et encyclopédies, témoins toujours en retard d'un usage » (p. 10) qui oscillent en permanence entre la tentation du consensus rassurant et celle du dissensus plus représentatif des aléas de la communication humaine. Pour Maurice Tournier: «Ouvrir le dictionnaire ne donne pas la solution. Selon le dictionnaire, sa date, les définitions peuvent changer. La neutralité du dictionnaire n'existe pas.» (p. 196.)

Ainsi, de la découverte de ces quelque 300 pages, écrites - n'en déplaise aux «puristes» - dans le respect des simplifications et rectifications orthographiques définies par le Conseil supérieur de la langue française et recommandées par l'Académie (voir le Journal officiel, $\mathrm{n}^{\circ}$ 100, 6 décembre 1990), le lecteur ressort secoué, dépoussiéré, revigoré. C'est un ouvrage à recommander à tous, car il bouscule avec intelligence un certain nombre de conventions, voire de convenances, et invite à un partage plurivoque de connaissances et d'idées au moment où la mondialisation et les risques d'uniformisation qu'elle comporte à tous les niveaux, en particulier celui de la pensée sociale et celui des modes d'expression, se posent de façon aiguë. En cela, la dernière phrase du livre: "Cette politisation et cette planétarisation des problèmes linguistiques est une caractéristique majeure de l'époque actuelle» (p. 306) peut être perçue à la fois comme un avertissement et comme un appel.

Éléonore Yasri-Labrique

Université de Montpellier 3, ARSER-DIPRALANG/EA 739

dipralang@univ-montp3.fr

Neologica. Revue internationale de néologie, $n^{\circ} 1$

John Humbley, Jean-François Sablayrolles éd.

2007, Paris, Garnier, 227 p.

Une nouvelle revue pour les mots, Neologica, dirigée par John Humbley et Jean-François Sablayrolles, est consacrée à l'analyse des néologismes et à l'étude théorique de la néologie. Bernard Quemada, qui signe l'avant-propos du premier numéro, éclaire le sens que prend la création de cette revue dans l'épistémologie des sciences du langage. Après «l'ostracisme de la linguistique théorisante des années 60-80 envers la lexicologie», la linguistique du 